\title{
Fc-fusion mimetics
}

Hanieh Khalili ${ }^{1,2}$, Peng T Khaw ${ }^{2}$ and Steve Brocchini ${ }^{1,2}$

${ }^{1}$ UCL School of Pharmacy, University College London, 29-39 Brunswick Square, London WC1N 1AX, UK

${ }^{2}$ NIHR Biomedical Research Centre, Moorfields Eye Hospital and UCL Institute of Ophthalmology, London, EC1V 9EL, UK 


\section{Abstract}

The Fc-fusion mimetic RpR $\underline{\mathbf{2}}$ was prepared by disulfide bridging conjugation using a PEG in the place of the Fc. RpR $\underline{\mathbf{2}}$ displayed higher affinity for VEGF than aflibercept caused primarily by a slower dissociation rate, which can prolong a drug at its site of action. RpRs have considerable potential for development as stable, organ specific therapeutics. 
To achieve effective bivalency and high affinity, the two Fabs in an IgG antibody are mobile and are linked together as if each Fab (or protein) is bound at the end of linear molecule (Figure 1). FpFs 1 are IgG antibody mimetics (Figure 1) designed to have enhanced stability and binding properties compared to lgGs. They are prepared from PEG-di(mono-sulfone) $\underline{\mathbf{3}}$ and two antibody fragments (Fabs). ${ }^{1}$ Fc-based fusion proteins $^{2}$ (Figure 1) are also capable of exploiting the therapeutic advantages of bivalency that are displayed by IgGs. Several Fc-fusion proteins are registered for clinical use ${ }^{3}$ and they will continue to offer considerable clinical potential because of Fc recycling, but they can be difficult to produce during early preclinical research and to scale for production. ${ }^{4} \mathrm{Fc}$-fusion proteins are also often prone to aggregation during downstream processing ${ }^{5}$ and have similar stability limitations as IgGs. There are therapeutic applications where the Fc is not needed or can cause problems. ${ }^{6}$ One area of interest is the use of antibody based medicines in organ specific applications such as the eye. In such cases, Fc recycling does not occur and effector function can be deleterious, especially in the treatment of inflammatory conditions. Improved stability is important to formulate more concentrated solutions to decrease the frequency of dose administration and improved binding properties such as slower dissociation rates are important for organ specific targeting. In an effort to further explore the potential of antibody-based mimetics that are made using the PEG-di(mono-sulfone) $\underline{\mathbf{3}}$ (Scheme $1 \mathrm{~A}$, Figure 1) we describe an Fc-fusion mimetic that we call RpR $\underline{\mathbf{2}}$, for receptor binding region-PEG-receptor binding region.

Aflibercept (Eylea) is a Fc-fusion protein that is used to treat age related macular degeneration (AMD) by binding to vascular endothelial growth factor (VEGF) in the back of the eye. It is administered by intravitreal injection directly into the eye. Fc-fusion proteins are related to IgG antibodies in that both have an Fc domain. IgG antibodies have two heavy and two light chains. The Fc-domain comprises the C2 and C3 regions of the two heavy chains. Interchain disulfides exist in IgG antibodies that are between the heavy chains in the hinge region to separate the Fc domain from the hinge and Fabs. Aflibercept is a homodimer that comprises 2 monomers of (VEGFR $1^{-}$ VEGFR $\left.{ }_{2}\right)-(C 2-C 3)$, referred to here as VEGFR 1 -VEGFR $2-F C \underline{\mathbf{5}}$ (Scheme 1B), with interchain disulfides expected to be in the peptide sequence between the target binding domains (VEGFR 1 -VEGFR ${ }_{2}$ ) and the Fc domain (C2-C3 regions) (Figure 1). ${ }^{7}$ Aflibercept is glycosylated with a total molecular weight of $115 \mathrm{kDa}$, of which $97 \mathrm{kDa}$ is due to the protein component. Each mono-sulfone moiety in reagent $\underline{\mathbf{3}}$ undergoes site-specific conjugation with the two cysteine thiols from an accessible disulfide by a sequence of addition-elimination reactions to insert a stable 3-carbon methylene bridge between the two thiols of the original disulfide (Scheme S1, ESI). ${ }^{8}$ The thiol 
ether bonds in a rebridged disulfide are more stable than the original disulfide bond.

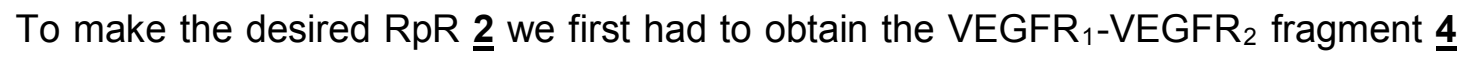
by proteolytic digestion of aflibercept to remove the Fc domain (Scheme 1B).

It was first confirmed that aflibercept migrated to an approximate molecular weight of about $115 \mathrm{kDa}$ by SDS PAGE (Figure 2, lane 1). Aflibercept was then treated with dithiothreitol (DTT) to reduce the accessible disulfides thought to exist in an hinge like region between the binding domain (VEGFR VEGFR $_{2}$ ) and the $F c$ domain. $A$ broad band appeared at $\sim 55-60 \mathrm{kDa}$ by SDS-PAGE (Figure 2, lane 2). We believe this band corresponds to the monomeric VEGFR ${ }_{1}-V_{E G F R}-F c \underline{\mathbf{5}}$ (Scheme 1B). Glycosylation is usually somewhat heterogeneous in therapeutic proteins, so we inferred that the broadness of the band at $\sim 55-60 \mathrm{kDa}$ was due to glycosylation heterogeneity. DDT was then removed using a PD-10 column and the reduced aflibercept solution was incubated with Ellman's reagent which indicated the presence of 4 accessible cysteine thiols in aflibercept (Figure 1S, Table 1S, ESI). This suggested that there are 2 cysteines in each VEGFR 1 -VEGFR $2-F c \underline{5}$ monomer which can form two disulfides in aflibercept analogous to what is found in the hinge region of $\lg G$ antibodies. Hence it was thought possible that an RpR $\underline{\mathbf{2}}$ derived from aflibercept could be prepared using the PEG-di(mono-sulfone) $\underline{\mathbf{3}}$. If only one cysteine had been present in the VEGFR ${ }_{1}$-VEGFR 2 -Fc $\underline{\mathbf{5}}$ monomer, there are stable, mono-thiol conjugation linkers available ${ }^{9}$ that would have been utilised in a bifunctional reagent analogous to PEG-di(mono-sulfone) $\underline{3}$.

Proteolytic digestion of aflibercept was then examined in an effort to obtain the monomeric VEGFR 1 -VEGFR ${ }_{2}$ fragment $\underline{4}$ (Scheme 1B). It was necessary that the cleavage should in aflibercept should occur to give the monomeric VEGFR 1 -VEGFR 2 fragment $\underline{4}$ to include the cysteines that form the accessible disulfides in aflibercept. Preliminary digestion studies of aflibercept using immobilised papain yielded only difficult to characterise small peptide fragments. We had previously used papain to digest IgGs to obtain Fabs to make FpFs, ${ }^{1,} 10$ but recognised that proteolytic digestion of different antibody subclasses and motifs can be difficult to control. ${ }^{11}$

A second proteolytic enzyme (IdeS enzyme, FabRICATOR ${ }^{\circledR}$, Genovis) that can cleave an $\lg G$ at glycine-glycine bonds ${ }^{12}$ in the hinge reagent to give $F(a b)_{2}$ was then examined. Incubation of aflibercept with IdeS resulted in 3 bands by SDS-PAGE (Figure 2, lane 3). New bands appeared at approximately $30 \mathrm{kDa}$ and 60-70 kDa. A third band at approximately $95-100 \mathrm{kDa}$ in this gel was thought to be undigested aflibercept. The lower molecular weight fragment at $30 \mathrm{kDa}$, which is thought to be the cleaved Fc, and the band for undigested aflibercept were removed by eluting the digestion mixture over a column that binds to the Fc domain (CaptureSelect Midispin, 
Genovis). This provided a purified a non-Fc containing fragment at 60-70 kDa (Figure 2, lane 4) which was thought to be the VEGFR ${ }_{1}-V_{E G F R}$ dimer $\underline{\mathbf{6}}$ (Scheme 1B).

Incubation of VEGFR 1 -VEGFR 2 dimer $\underline{\mathbf{6}}$ with DTT caused this fragment to disappear to give 2 lower molecular weight fragments (Figure 2, lane 5). These fragments are thought to be the desired VEGFR ${ }_{1}-V_{E G F R}$ monomer $\underline{4}$ (Scheme 1). Two bands are often observed after reduction of Fabs that are obtained by proteolytic digestion. This is often thought to be due to miscleavage reactions during proteolysis. This may be exacerbated with aflibercept due to structural differences with IgGs and aflibercept glycosylation. There are five $\mathrm{N}$-glycosylation sites on each monomeric VEGFR $_{1}-V_{E G F R}-F c$ fragment $\underline{\mathbf{5}}$ which may be partially or completely glycosylated. There may also be additional heterogeneity caused by differences in saccharide structure in the glycosylated protein.

To prepare the RpR $\underline{\mathbf{2}}$, the VEGFR $\mathrm{V}_{1}-\mathrm{VEGFR}_{2}$ dimer $\underline{\mathbf{6}}$ was first incubated with DTT for 30 minutes to give the VEGFR 1 -VEGFR 2 monomer $\underline{4}$ (Scheme $1 \mathrm{~B}$ ). The reaction mixture was carefully eluted over a PD-10 column to remove the DTT while avoiding disulfide reformation, and then the PEG di(mono-sulfone) reagent $\underline{3}$ (derived from a $10 \mathrm{kDa} P E G$ precursor) was added to the solution of the monomeric VEGFR 1 $\mathrm{VEGFR}_{2} \underline{4}$. Incubation of the reaction mixture for $3 \mathrm{~h}$ (Figure $2 \mathrm{~S}$, lane $1, \mathrm{ESI}$ ) was then followed by purification by size exclusion chromatography (Figure 2S, lanes 2-10, ESI) to give the purified RpR $\underline{\mathbf{2}}$ which appeared in a band at approximately $70 \mathrm{kDa}$ (Figure 2, lanes 6 and 7). Two detection dyes were used, first coomassie blue to detect protein (lane 6) and then barium iodide to detect the PEG (lane 7) being conjugated to the protein. Starting from $0.8 \mathrm{mg}$ (in $1.0 \mathrm{~mL}$ ) of VEGFR -VEGFR $_{2}$ dimer $\underline{\mathbf{6}}$, approximately $0.16 \mathrm{mg}$ (in $0.5 \mathrm{~mL}$ ) of RpR $\underline{2}$ was obtained ( $20 \%$ yield).

At $25^{\circ} \mathrm{C}$ the purified $\mathrm{RpR} \underline{\mathbf{2}}$ displayed a solution size of $10.7 \pm 0.5 \mathrm{~nm}(\mathrm{Pd}, 0.7$ $\pm 0.1 \mathrm{~nm})$, which is similar to the starting aflibercept $(10.2 \pm 0.7 \mathrm{~nm} ; \mathrm{Pd}, 0.6 \pm 0.1 \mathrm{~nm})$. The FpF antibody mimetics 1 were also a similar solution size to the corresponding $\operatorname{lgG} .{ }^{1}$ This is in stark contrast to when PEG is conjugated only at one terminus to a single protein where the solution size of a PEG-protein conjugate is dominated by the random coil nature of $P E G .{ }^{13}$ When only one terminus of $P E G$ is conjugated to a protein, the other PEG terminus has considerable freedom to allow the PEG to maintain a large solution structure.

As macromolecules, both $\mathrm{FpF}$ and $\mathrm{RpR}$ structures have proteins at each terminus of the PEG scaffold which would constrain the motion of the PEG, so these antibody mimetics are analogous to A-B-A block copolymers where it is known that linear polymers functionalised at both ends can self-associate. ${ }^{14}$ The dimeric VEGFR 1 VEGFR ${ }_{2}$ fragment $\underline{\mathbf{6}}$ also displayed a solution size of $10.03 \pm 0.1 \mathrm{~nm}(\mathrm{Pd}, 0.7 \pm 0.1$ 
$\mathrm{nm}$ ) which is similar to both aflibercept and RpR 2. Interestingly, when the VEGFR VEGFR ${ }_{2}$ fragment $\underline{\mathbf{6}}$ was treated with DTT and the cysteine thiols were blocked with iodoacetamide, the cysteine thiol-capped monomeric VEGFR ${ }_{1}-V_{E G F R}$ fragment $\underline{7}$ (Scheme 1B) displayed a solution size of $7.2 \pm 0.4 \mathrm{~nm}(\mathrm{Pd}, 0.7 \pm 0.1 \mathrm{~nm})$. Although the dimer $\underline{\mathbf{6}}$ is twice the molecular weight of the monomer $\underline{\mathbf{7}}$, its solution size is only about $40 \%$ larger suggesting that there is intramolecular association of the VEGFR - $^{-}$ VEGFR 2 domains within the dimer $\underline{\mathbf{6}}$.

The binding properties of the RpR $\underline{\mathbf{2}}$ and aflibercept were then evaluated by surface plasmon resonance (Biacore) to determine the affinity $\left(\mathrm{K}_{\mathrm{D}}\right)$, and the rate constants of association $\left(k_{a}\right)$ and dissociation $\left(k_{d}\right)$ (Table 1). Vascular endothelial growth factor-165 (VEGF ${ }_{165}$ ), which is a ligand for aflibercept, was immobilised at a density to minimise or prevent rebinding events (91 RU). ${ }^{1,15}$ The dissociation rate $\left(k_{d}\right)$ for the RpR $\underline{\mathbf{2}}$ was slower than what was observed with aflibercept. Interestingly, the $\mathrm{k}_{\mathrm{a}}$ appeared to be slightly faster in RpR $\underline{\mathbf{2}}$ compared to aflibercept. This is in contrast to what was previously observed for anti-VEGF FpF which had a slower association rate than the precursor $\lg G$ antibody. ${ }^{1}$ However it was the decreased $k_{d}$ of $R p R \underline{\mathbf{2}}$ that appeared to be the dominating factor to cause the improved affinity of $R p R \underline{\mathbf{2}}$ compared to aflibercept (Table 1). Representative fitting curves for aflibercept and RpR $\underline{\mathbf{2}}$ are shown in the ESI (Figure S3, ESI).

Exploiting reduced dissociation rates may be a viable strategy to increase efficacy by increasing the residence time and mode of action within specific tissue. ${ }^{16}$ Although the reduction in $\mathrm{k}_{d}$ for $\mathrm{FpF} \underline{\mathbf{1}}$ is also slower than the parent $\operatorname{lgG},{ }^{1}$ there appears to be a greater relative reduction in $k_{d}$ for the RpR $\underline{2}$ compared to its parent Fc-fusion (i.e. aflibercept). During initial dissociation steps from the ligand of one of the two VEGFR ${ }_{1}$-VEGFR 2 domains in the RpR $\underline{2}$, PEG conformational flexibility may be more efficient for rebinding than the polypeptide linking the Fc domain to the VEGFR - $^{-}$ VEGFR ${ }_{2}$ domain in aflibercept. This suggests there is less flexibility in the bivalent binding moieties in the Fc-fusion protein (aflibercept) than there is in an lgG (e.g. bevacizumab).

The VEGF binding of the capped VEGFR ${ }_{1}-V_{E G F R}$ monomer $\underline{7}$ (Figure S4, ESI) was reduced when compared to the VEGFR 1 -VEGFR ${ }_{2}$ dimer $\underline{\mathbf{6}}$. This exemplified the advantages of the cooperative bivalent binding that is possible with (i) aflibercept, (ii) the dimeric VEGFR 1 -VEGFR 2 fragment $\underline{\mathbf{6}}$ and (iii) RpR $\underline{\mathbf{2}}$ (Table 1). The similar binding properties that were observed for both the dimer 6 and aflibercept suggests that the placement of the accessible disulfides linking each monomer in aflibercept is important for the mobility of the VEGFR ${ }_{1}$-VEGFR 2 binding domains. Inclusion of a polypeptide sequence to extend the VEGFR 1 -VEGFR 2 receptor domains away from 
the aflibercept disulfides to better optimise dissociation rates would be expected to make aflibercept less stable. Such an added polypeptide sequence to increase the flexibility of the VEGFR 1 -VEGFR 2 receptor domains would invariably lack secondary structure in a similar way to the hinge region of $\lg G$ antibodies. While the hinge region in IgG antibodies provides the flexibility needed for cooperative and bivalent binding of both Fabs, ${ }^{17}$ the lgG hinge region is also vulnerable to degradation and disulfide scrambling. ${ }^{18}$ The stable conjugation imparted by PEG-di(mono-sulfone) $\underline{\mathbf{3}}$ and use of a PEG scaffold provides enough flexibility of the VEGFR 1 -VEGFR $R_{2}$ binding moieties to potentially maximise both association and dissociation rates that could be important in the development of new therapeutics.

$\mathrm{RpR} \underline{\mathbf{2}}$ was then evaluated in vitro using a human umbilical vein endothelial cell (HUVEC) co-culture (Figure 3). This assay measures the migration and the formation of an anastomosing network that is characterised by tubule and junction formation during HUVEC proliferation. These processes are characteristic for angiogenesis and are often a good in vitro measurement for angiogenesis. ${ }^{19} \mathrm{RpR} \underline{\mathbf{2}}$ and aflibercept were incubated with VEGF ${ }_{165}$ at different molar ratios of $3.0,1.5,0.5$ for 2 hours at $37^{\circ} \mathrm{C}$ prior to incubation with HUVECs. VEGF 165 and anti-mouse TNF- $\alpha$ IgG were used for positive controls. Images were obtained after fixing HUVECs with an anti-CD31 antibody to differentiate between the endothelial tubular network and non-endothelial structures of similar apparent morphology (Figure 3A). These images suggest that both aflibercept and the RpR $\underline{\mathbf{2}}$ have similar anti-angiogenic properties. Quantification of tubule (Figure S5, ESI) and junction formation (Figure 3B) (AngioSys Image Analysis Software, TCS Cellworks Ltd.) showed that the formation of these structures were similarly inhibited in a concentration dependent manner by both RpR $\underline{\mathbf{2}}$ and aflibercept.

To summarise, a new antibody Fc-fusion mimetic called an RpR was prepared. Aflibercept is a clinically used Fc-fusion protein that targets VEGF was used for these studies. Proteolytic digestion of aflibercept followed by incubation with DTT provided the monomeric VEGFR ${ }_{1}$-VEGFR 2 domain $\underline{4}$ that was then conjugated to the PEG$\mathrm{di}($ mono-sulfone) $\underline{\mathbf{3}}$ by disulfide bridging conjugation to give the anti-VEGF RpR $\underline{\mathbf{2}}$. The strategy to proteolytically digest aflibercept provided the means to compare the properties of the RpR Fc-fusion mimetic $\underline{\mathbf{2}}$ with aflibercept which is an existing, clinically used Fc-fusion protein. The solution size of RpR $\underline{\mathbf{2}}$ and its in vitro activity are comparable to aflibercept. Of most interest is that binding studies show that $R p R \underline{2}$ has higher affinity for VEGF compared to aflibercept primarily due to a slower dissociation rate. Antibody based mimetics such as RpR $\underline{\mathbf{2}}$ have considerable potential for development as stable, organ specific therapeutics. 
We are thankful for funding from the National Institute of Health Research (NIHR) Biomedical Research Centre at Moorfields Eye Hospital NHS Foundation Trust and UCL Institute of Ophthalmology, Moorfields Special Trustees, the Helen Hamlyn Trust (in memory of Paul Hamlyn), Medical Research Council, Fight for Sight and Freemasons Grand Charity. SB is also grateful for funding from the UK En- gineering \& Physical Sciences Research Council (EPSRC) for the EPSRC Centre for Innovative Manufacturing in Emergent Macromolecular Therapies. Financial support from the consortium of industrial and governmental users for the EPSRC Centre is also acknowledged. 


\section{References}

1. H. Khalili, A. Godwin, J. W. Choi, R. Lever, P. T. Khaw, and S. Brocchini, Bioconjugate Chem., 2013, 24, 1870-1882.

2. (a) S. M. Chamow, T. Ryll, H. B. Lowman, and D. Farson, eds. Therapeutic FCFusion Proteins. Vol. 10. 2013, Wiley Blackwell: Weinheim. 419; (b) D. M. Czajkowsky, J. Hu, Z. Shao, and R. J. Pleass, EMBO Mol. Med., 2012, 4, 10151028.

3. W. R. Strohl, BioDrugs 2015, 29, 215-239.

4. (a) A. B. V. Spriel, H. H. V. Ojik, and J. G. J. Winkel, Immunol. Today 2000, 21, 391-398; (b) H. R. Hoogenboom, Nat. Biotechnol., 1997, 15, 125-126.

5. (a) A. A. Cordes, C. W. Platt, J. F. Carpenter, and T. W. Randolph, J. Pharm. Sci., 2012, 101, 1400-1409; (b) M. C. Manning, D. K. Chou, B. M. Murphy, R. W. Payne, and D. S. Katayama, Pharm. Res., 2010, 27, 544-575.

6. (a) M. Kolfschoten, J. Schuurman, M. Losen, W. K. Bleeker, P. Martinez, E. Vermeulen, T. H. D. Bleker, D. Wiegman, T. Vink, L. A. Aarden, M. H. D. Baets, J. Winkel, R. C. Aalberse, and P. Parren, Science 2007, 13, 1554-1557; (b) S. Murinello, R. F. Mullins, A. J. Lotery, V. H. Perry, and J. L. Teeling, Invest. Ophthalmol. Vis. Sci., 2014, 55, 247-258; (c) A. L. Nelson, MAbs, 2010, 2, 7783; (d) A. Chapman, P. Antoniw, M. Spitali, S. West, S. Stephens, and D. King, Nat. Biotechnol., 1999, 17, 780-783.

7. (a) EMA (European Medicines Agency), Assessment report for aflibercept (EMA/646256/2012), European Medicines Agency Committee for Medicinal Products for Human Use, Editor. 2012. p. 83; (b) K. K. Ciombor, J. Berlin, and E. Chan, Clin. Cancer. Res., 2013, 19, 1920-1925; (c) J. Holash, S. Davis, N. Papadopoulos, S. D. Croll, L. Ho, M. Russell, P. Boland, R. Leidich, D. Hylton, E. Burova, E. loffe, T. Huang, C. Radziejewski, K. Bailey, J. P. Fandl, T. Daly, S. J. Wiegand, G. D. Yancopoulos, and J. S. Rudge, Proc. Natl. Acad. Sci. U $S$ A, 2002, 99, 11393-11398.

8. (a) S. Shaunak, A. Godwin, J. W. Choi, S. Balan, E. Pedone, D. Vijayarangam, S. Heidelberger, I. Teo, M. Zloh, and S. Brocchini, Nat. Chem. Biol., 2006, 2, 312-313; (b) S. Balan, J. W. Choi , A. Godwin, I. Teo, C. M. Laborde, S. Heidelberger, M. Zloh, S. Shaunak, and S. Brocchini, Bioconjugate Chem., 2007, 18, 61-76.

9. G. Badescu, P. Bryant, J. Swierkosz, F. Khayrzad, E. Pawlisz, M. Farys, Y. Cong, M. Muroni, N. Rumpf, S. Brocchini, and A. Godwin, Bioconjugate Chem., 2014, 25, 460-469.

10. H. Khalili, A. Godwin, J. Choi, R. Lever, and S. Brocchini, Bioconjugate Chem., 2012, 23, 2262-2277.

11. (a) M. G. Mage, Methods Enzymol., 1980, 70, 142-150; (b) P. Parham, J. Immunol., 1983, 131, 2895-2902.

12. (a) B. P. Johansson, O. Shannon, and L. Bjorck, PLoS One, 2008, 3, 1-6; (b) P. Åkesson, L. Moritz, M. Truedsson, B. Christensson, and U. V. PawelRammingen, Infection and Immunity 2006, 74, 497-503.

13. C. Fee, Biotechnol. Bioeng., 2007, 98, 725-731.

14. (a) F. Lo Verso and C. N. Likos, Polymer, 2008, 49, 1425-1434; (b) A. N. Semenov, J. F. Joanny, and A. R. Khokhlov, Macromolecules, 1995, 28, 10661075.

15. J. Yang, X. Wang, G. Fuh, L. Yu, E. Wakshull, M. Khosraviani, E. S. Day, B. Demeule, J. Liu, S. J. Shire, N. Ferrara, and S. Yadav, Mol. Pharm., 2014, 11, 3421-3430.

16. G. Vauquelin and S. J. Charlton, Br. J. Pharmacol., 2010, 161, 488-508.

17. (a) W. Paul, ed. Fundamental immunology. 2012, Lippincott Williams and Wilkins. . 140-142; (b) L. J. Harris, S. B. Larson, E. Skaletsky, and A. 
Mcpherson, Immunol. Rev. , 1998, 163, 35-43; (c) X. Wang, S. Kumar, and S. Singh, Pharm. Res., 2011, 28, 3128-3143.

18. (a) J. Vlasak and R. Ionescu, MAbs, 2011, 3, 253-263; (b) B. Yan, D. Boyd, T. Kaschak, J. Tsukuda, A. Shen, Y. Lin, S. Chung, P. Gupta, A. Kamath, A. Wong, J.-M. Vernes, G. Y. Meng, K. Totpal, G. Schaefer, G. Jiang, B. Nogal, C. Emery, M. Vanderlaan, P. Carter, R. Harris, and A. Amanullah, J. Biol. Chem., 2012, 287, 5891-5897.

19. Y. Wang, D. Fei, M. Vanderlaan, and A. Song, Angiogenesis, 2004, 7, 335-345. 


\section{Figure Legends}

Figure 1. IgG and Fc fusion proteins and their respective mimetics, FpF $\underline{1}$ and $\mathrm{RpR} \underline{\mathbf{2}}$.

Figure 2. SDS-PAGE gels of VEGFR ${ }_{1}-\mathrm{VEGFR}_{2}$ dimer $\underline{\mathbf{6}}$ obtained by the proteolytic digestion of aflibercept and preparation of RpR $\underline{\mathbf{2}}$. Novex Bis-Tris $4-12 \%$ gel stained with colloidal blue for protein and barium lodide for PEG (lane 7). M: standard protein markers, Lane 1: aflibercept, Lane 2: aflibercept treated with DTT to give VEGF ${ }_{1}$ VEGF $_{2}-F_{c}$ monomer $\underline{\mathbf{5}}$, Lane 3: aflibercept-Ides digestion mixture, Lane 4: VEGFR ${ }_{1}$ VEGFR ${ }_{2}$ dimer $\underline{\mathbf{6}}$, Lane 5: VEGFR 1 -VEGFR ${ }_{2}$ monomer $\underline{\mathbf{4}}$, Lanes 6, 7: purified RpR $\underline{\mathbf{2}}$.

Figure 3. (A) Representative images that were used for AngioSys analysis to quantitate tubule formation using a HUVEC-fibroblast angiogenesis assay. The dark structures (tubules) are indicative of angiogenesis and were analysed to determine the number of junctions. (B) Number of junctions observed for medium alone, medium + VEGF, Anti-mouse TNF-a IgG + VEGF, aflibercept + VEGF and RpR + VEGF. Ratios are the amount of test compound to VEGF. VEGF was present at a fixed concentration of $10 \mathrm{ng} / \mathrm{mL}$. Data are expressed as the mean of three individual cultures per treatment environment.

\section{Scheme Legend}

Scheme 1. (A) Preparation RpR $\underline{\mathbf{2}}$ from PEG-di(mono-sulfone) $\underline{\mathbf{3}}$ and two equivalents of the VEGFR 1 -VEGFR 2 monomer 4._(B) Use of aflibercept to obtain the monomeric $\mathrm{VEGF}_{1}-\mathrm{VEGF}_{2} \underline{4}$ and $\mathrm{VEGF}_{1}-\mathrm{VEGF}_{2}-\mathrm{Fc} \underline{\mathbf{5}}$ fragments. Proteolytic digestion of aflibercept with the IdeS enzyme results in the cleavage of the Fc to give the VEGFR - $^{-}$ VEGFR ${ }_{2}$ dimer $\underline{\mathbf{6}}$ that after treatment with DTT gives the VEGFR ${ }_{1}-V_{E G F R}$ monomer $\underline{4}$ which was used to make RpR $\underline{2}$. The VEGFR VVEGFR $_{2}$ monomer $\underline{4}$ was also incubated iodoacetamide to give the thiol capped VEGFR 1 -VEGFR $R_{2}$ fragment $\underline{\mathbf{Z}}$ for binding studies.

\section{Table Legend}

Table 1. Binding kinetic constant rate of aflibercept, VEGFR ${ }_{1}-V_{E G F R}$ dimer $\underline{\mathbf{6}}$ and RpR $\underline{\mathbf{2}}$, Affinity, dissociation and association rate constants were studied using CM3 chip immobilised with $91 \mathrm{RU}$ human VEGF at $25^{\circ} \mathrm{C}$. The range of concentrations used for the conjugates was $0.06 \mu \mathrm{M}$ to $1.6 \mu \mathrm{M}$. Data were collected from 2 independent experiments for RpR $\underline{2}$ and 3 independent experiments for aflibercept and VEGFR ${ }_{1}$ VEGFR ${ }_{2}$ dimer $\underline{\mathbf{6}}$. 
Figures

Figure 1

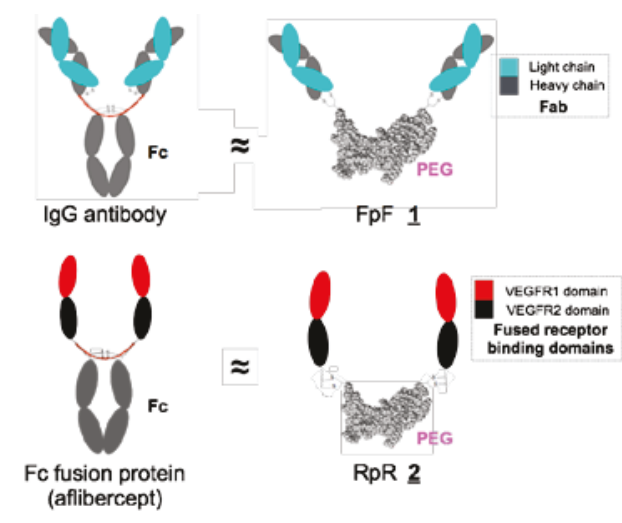

Figure 2

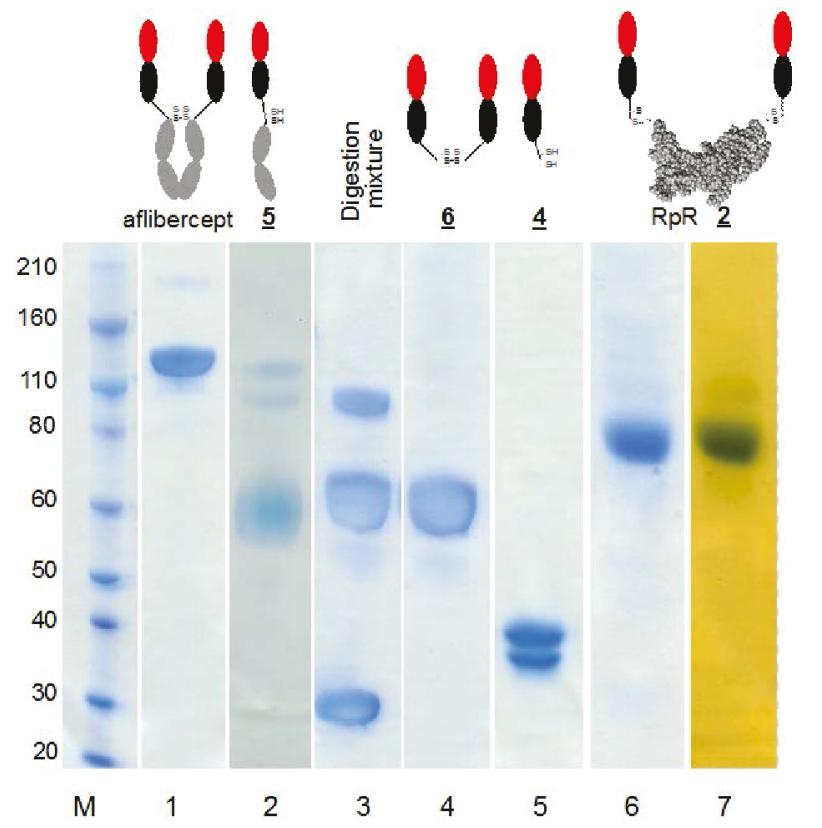




\section{Figure 3}
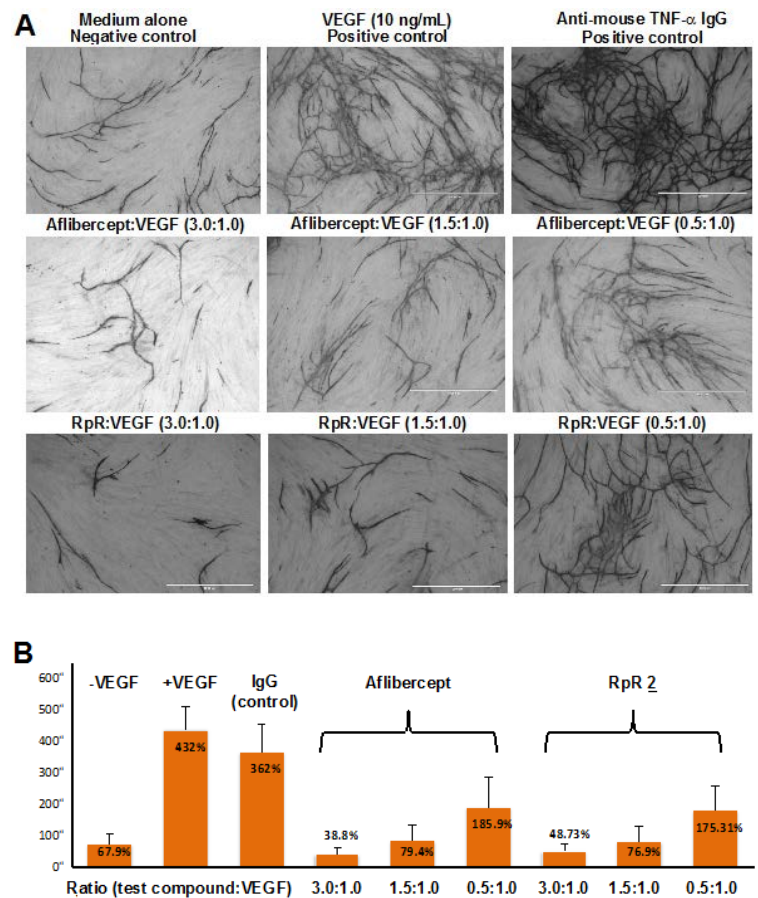


\section{Scheme 1}

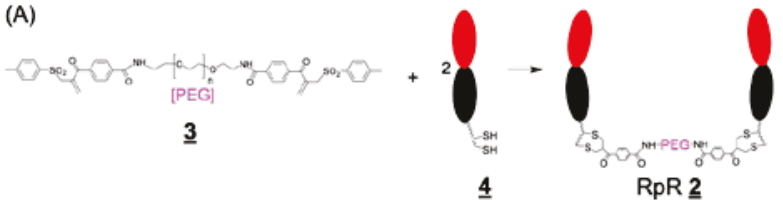

(B)

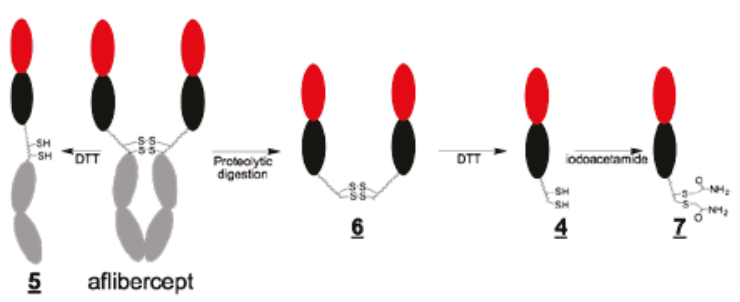

Table 1

\begin{tabular}{cccc}
\hline Sample & $\begin{array}{c}\mathbf{K}_{\mathbf{a}} \\
\left(\times 10^{5}\right) \mathbf{~ M}^{-1} \mathbf{s}^{-1}\end{array}$ & $\begin{array}{c}\mathbf{k}_{\mathbf{d}} \\
\left(\times 10^{-4}\right) \mathbf{~ s}^{-1}\end{array}$ & $\begin{array}{c}\mathbf{K}_{\mathbf{D}} \\
\left(\mathbf{k}_{\mathbf{d}} / \mathbf{k}_{\mathbf{a}}\right) \mathbf{n M}\end{array}$ \\
\hline Aflibercept & 0.88 & 4.20 & 4.78 \\
$\left(\text { VEGFR }_{1}-V_{E G F R}\right)_{2} \underline{\mathbf{6}}$ & 1.20 & 5.20 & 4.30 \\
RpR $\underline{\mathbf{2}}$ & 1.13 & 1.90 & 1.71 \\
\hline
\end{tabular}

